\title{
BMJ Open Evaluating the role of paternal factors in aetiology and prognosis of recurrent pregnancy loss: study protocol for a hospital-based multicentre case-control study and cohort study (REMI III project)
}

\author{
Nadia du Fossé (D) , Marie-Louise van der Hoorn, ${ }^{1}$ Michael Eikmans, ${ }^{2}$ \\ Sebastiaan Heidt, ${ }^{2}$ Saskia le Cessie, ${ }^{3,4}$ Annemarie Mulders, ${ }^{5}$ Jan van Lith, ${ }^{1}$ \\ Eileen Lashley ${ }^{1}$
}

To cite: du Fossé $\mathrm{N}$, van der Hoorn M-L, Eikmans M, et al. Evaluating the role of paternal factors in aetiology and prognosis of recurrent pregnancy loss: study protocol for a hospital-based multicentre case-control study and cohort study (REMI III project). BMJ Open 2019;9:e033095. doi:10.1136/ bmjopen-2019-033095

- Prepublication history for this paper is available online. To view these files, please visit the journal online (http://dx.doi. org/10.1136/bmjopen-2019033095).

Received 19 July 2019 Revised 08 September 2019 Accepted 14 October 2019

Check for updates

(C) Author(s) (or their employer(s)) 2019. Re-use permitted under CC BY-NC. No commercial re-use. See rights and permissions. Published by BMJ.

For numbered affiliations see end of article.

Correspondence to

Nadia du Fossé;

n.a.du_fosse@lumc.n

\section{ABSTRACT}

Introduction Recurrent pregnancy loss (RPL) is defined as the spontaneous demise of two or more pregnancies before the fetus reaches viability. Despite investigation of multiple known maternal risk factors, in more than $50 \%$ of couples, this condition remains unexplained. Studies focusing on paternal factors in RPL are scarce, and therefore, paternal evaluation in RPL is currently very limited. However, regarding single miscarriage, there are multiple publications suggesting a contributive role of paternal factors. In this project, we aim to identify paternal factors associated with RPL and to improve couple-specific prediction of future pregnancy outcomes by developing a prediction model containing both maternal and paternal factors.

Methods and analysis In a case-control design, the relation between unexplained RPL and paternal age, lifestyle factors, sperm DNA damage and immunomodulatory factors in peripheral blood and semen will be studied. Prospectively, 135 couples with naturally conceived unexplained RPL (cases) and 135 fertile couples without a history of pregnancy loss (controls) will be included, with collection of paternal blood and semen samples and documentation of clinical and lifestyle characteristics. In addition, 600 couples from both groups will be included retrospectively. To adjust for confounders, multivariate logistic regression will be used. The predictive value of paternal and maternal factors will be studied in the total RPL cohort consisting of approximately 735 couples. The primary outcome of the cohort study is live birth within 5 years after initial visit of the clinic. Secondary outcomes are ongoing pregnancy, time interval until next pregnancy and pregnancy complications.

Ethics and dissemination This project is approved by the Medical Research Ethics Committee of the Leiden University Medical Center. No risks or burden are expected from the study. The findings of this study will be disseminated via peer-reviewed publications and presentations at international conferences.

Trial registration number NL7762
Strengths and limitations of this study

- First large multicentre prospective study to investigate the contribution of both paternal lifestyle and biological factors to the development of recurrent pregnancy loss (RPL).

- Extensive cohort of RPL couples that will provide sufficient data to develop a multivariable prediction model for future pregnancy outcomes.

- Generalisability of the outcomes is increased by the collaboration between two Dutch tertiary centres in different regions that serve a diverse patient population.

- Control of bias by adjustment for important maternal confounders, to investigate the independent effect of paternal factors on RPL.

- Observational studies on lifestyle factors are prone to response and recall bias; a potential limitation of this study.

\section{INTRODUCTION}

Spontaneous pregnancy loss is the most common complication in human pregnancy, defined as the loss of conception before the fetus reaches viability $(<24$ weeks of gestation) and occurs in $10 \%-15 \%$ of clinically recognised pregnancies. ${ }^{12}$ Pregnancy loss is also often referred to as miscarriage; however, this term is recommended to be used for confirmed intrauterine pregnancy losses only. ${ }^{3}$ Recurrent pregnancy loss (RPL) is defined as two or more losses in one couple. ${ }^{1}$ This condition affects approximately $1 \%-3 \%$ of all couples of reproductive age. ${ }^{45}$

RPL is a highly heterogeneous condition. Among the multifaceted risk factors are maternal acquired thrombophilia 
(antiphospholipid syndrome), structural uterine abnormalities, thyroid autoimmunity and parental balanced chromosomal translocations. ${ }^{6-12}$ Maternal age is a strong risk factor for pregnancy loss, mainly based on the increased prevalence of the fetal aneuploid abnormalities with advancing age. ${ }^{13}$ Maternal lifestyle factors, such as smoking, alcohol and caffeine consumption and adiposity, are also associated with RPL. ${ }^{14-19}$

Despite extensive investigations, a potential underlying condition cannot be identified in $50 \%-70 \%$ of couples that present with RPL. ${ }^{20}$ Limited understanding of underlying pathophysiological mechanisms means that options for effective interventions are lacking. Currently, no evidence-based therapeutic options are available for couples with unexplained RPL. Clinical management is either empirical or primarily focused on providing supportive care, which has been shown to have a beneficial effect. ${ }^{22}$ Part of this supportive care is counselling on the prognosis and success rate of subsequent pregnancies in couples with RPL. Lund et al evaluated the prognosis of 987 women with RPL and found that $67 \%$ achieved a live birth within 5 years after first consultation. ${ }^{23}$ They showed that the chance of at least one subsequent live birth decreased significantly with increasing maternal age and cumulative number of preceding miscarriages. Other studies reported live birth rates ranging from 57\% to $95 \% .^{24-26}$ This large variation might be explained by the use of different definitions for RPL (2 vs 3 losses, consecutive vs non-consecutive, primary vs secondary), by the degree of monitoring of the women and by inclusion or exclusion of biochemical pregnancies in the definition of RPL. ${ }^{23}$ Nevertheless, these results demonstrate that although unidentified factors increasing the risk for pregnancy loss may exist, they do not necessarily prevent the development of a successful pregnancy. An essential part of the management of couples with RPL is to give trustworthy advice on the prognosis for a next pregnancy. However, the main limitation in current prognostic studies on unexplained RPL is the lack of adjustment for relevant risk factors, disabling the possibility of individual risk estimation. ${ }^{23} 27$

The investigation of paternal contribution to RPL is currently limited to exploring the male karyotype. When considering counselling at an individual level, paternal factors may be included to establish a couple specified prognosis. Since the oocyte and the spermatozoon contribute equally to the genome of the embryo, it is biologically plausible to think that part of the idiopathic RPL cases could be explained by paternal factors. Some studies have evaluated the effect of paternal risk factors such as age, smoking and somatic health factors on the development of miscarriages, though these studies are mostly restricted to single miscarriage or to couples undergoing assisted reproductive techniques (ART) ${ }^{28-30}$ Following the absence of a consistent association between conventional semen parameters and RPL, ${ }^{31-38}$ the majority of recent studies addressing paternal factors and pregnancy losses focused on genetic defects, with sperm DNA fragmentation showing the most promising results. Both Robinson $e t a l^{39}$ and Zhao $e t a l^{40}$ showed in a meta-analysis that a high level of sperm DNA damage is associated with an increased miscarriage rate after in vitro fertilisation/ intracytoplasmic sperm injection (IVF/ICSI) treatment. Two other recent meta-analyses found an increased mean difference in sperm DNA fragmentation of $12 \%$ in male partners of women with RPL compared with men whose partners had successful pregnancies. ${ }^{41} 42$ However, prospective studies in RPL couples evaluating the predictive value of sperm DNA fragmentation on future pregnancy outcomes are lacking.

In addition, imbalances in seminal immunomodulatory factors may contribute to the development of RPL. During pregnancy, the maternal immune system has to tolerate the presence of semiallogeneic cells in maternal tissue. Seminal fluid contains various signalling molecules that are thought to induce lymphocyte proliferation, affect natural killer cell activity and modify cytokine release from antigen presenting cells, resulting in tolerance towards paternal alloantigens. ${ }^{43-45}$ An optimal balance of proinflammatory and immunomodulatory factors seems to be necessary for the induction of immunological tolerance and the process of implantation and placentation. ${ }^{46}$ Increased plasma levels of interleukin-18 (IL-18) and IL-8 and decreased levels of IL-11 were found to be negatively correlated to fertilisation and implantation. ${ }^{47} 48$ In subfertile couples with normospermia, including a small subgroup with a history of RPL, decreased concentrations of IL- $1 \beta$ and increased interferon- $\gamma$ (IFN- $\gamma$ ) were present in the seminal plasma. ${ }^{49}$ The same study also suggests a correlation between levels of proinflammatory and antiinflammatory cytokines in paternal peripheral blood and reproductive outcome. In case of such correlations, cytokine micropatterns in blood serum could serve as a proxy for those in the seminal plasma and could potentially be suitable as easily available prognostic markers in clinical practice. However, larger prospective studies are required to assess this.

In this study, we hypothesise that unexplained RPL is an issue stemming from both the female and the male. Our overall aims are to identify paternal factors that are associated with the development of this condition and to assess the predictive value of these factors for future reproductive outcomes in couples with RPL, in addition to maternal factors.

\section{STUDY OBJECTIVES \\ Primary objectives}

To identify paternal factors that are associated with unexplained RPL.

Paternal factors that will be assessed are: age, smoking, alcohol intake, recreational drugs intake, caffeine intake, body mass index (BMI), level of sperm DNA fragmentation and immunomodulatory factors in seminal plasma and paternal peripheral blood. 


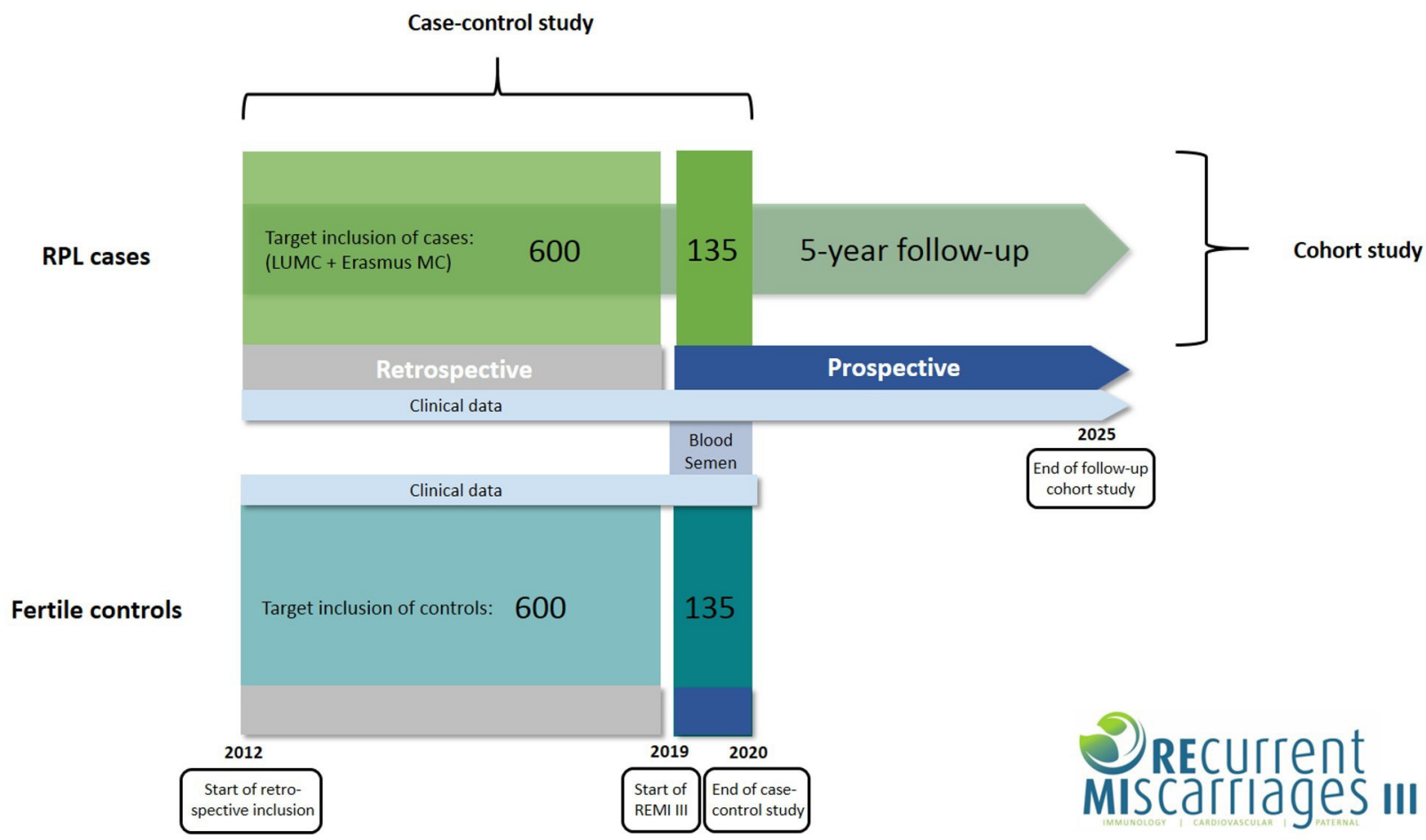

Figure 1 Schematic diagram of study design. For the case-control study the target for inclusion is 735 couples in each arm. Of these 735 couples, 600 will be included retrospectively (2012-2018) and 135 will be included prospectively (2019-2020). Semen and blood will be collected from prospectively included men only. Couples with RPL (cases) are also part of a cohort study. We aim to complete a 5-year follow-up of these couples, starting from their individual point of inclusion. Control couples will not be in follow-up. LUMC, Leiden University Medical Center; MC, Medical Center; REMI, REcurrent Mlscarriages; RPL, recurrent pregnancy loss.

To assess the correlation between level of sperm DNA fragmentation and immunomodulatory factors in seminal plasma and paternal peripheral blood.

\section{Secondary objectives}

To assess the prognostic effect of paternal factors on reproductive outcomes in couples with unexplained RPL.

To develop a prediction model containing both maternal and paternal factors to predict the chance of a successful pregnancy for couples with unexplained RPL.

\section{METHODS AND ANALYSIS \\ Study design}

The primary objectives are focused on aetiology and will be addressed in a case-control study. In this case-control study, paternal factors are compared between couples with RPL and control couples. The expected duration of the case-control study is 1 year.

The secondary objectives will be addressed in a retrospective and prospective cohort study of couples with RPL. For all couples, participating in the cohort study, we aim to complete a follow-up on pregnancy outcomes of 5 years after first consultation.

A schematic overview of the study design is shown in figure 1.

\section{Eligibility criteria}

Inclusion criteria for RPL couples are:
- Unexplained RPL

According to the European Society of Human Reproduction and Embryology (ESHRE) RPL guideline ${ }^{1}$ defined as the loss of $\geq 2$ pregnancies in the current relationship, without any of the following known risk factors: parental chromosomal abnormalities, uterine abnormalities, acquired thrombophilia and thyroid autoimmunity. The definition includes all pregnancy losses before 24th week of gestation verified by ultrasonography or uterine curettage and histology and also non-visualised pregnancies (including biochemical pregnancy loss and/or resolved and treated pregnancies of unknown location) verified by positive urine or serum hCG. If identified as such, ectopic and molar pregnancies are not included. Pregnancy losses do not need to be consecutive.

Exclusion criteria for RPL couples are:

- Known risk factors for RPL as defined above.

- Mental or legal incapability of either the male or female.

- Pregnancy after ART.

- Pregnancy after oocyte, embryo or spermatozoa donation.

- Loss of $<2$ pregnancies in the current relationship.

Inclusion criteria for control couples are:

- Proven fertility (ie, pregnant at the time of inclusion or previously experienced pregnancy in the same relationship). 
Exclusion criteria for control couples are:

- Previous spontaneous pregnancy loss.

- One of the following conditions: parental chromosomal abnormalities, uterine abnormalities, acquired thrombophilia and thyroid autoimmunity (this will not be investigated, however, couples are excluded when it is known).

- Mental or legal incapability of either the male or female.

- Pregnancy after ART.

- Pregnancy after oocyte, embryo or spermatozoa donation.

\section{Study population and recruitment}

Couples with RPL that visit the RPL outpatient clinic of Leiden University Medical Center (LUMC) or early pregnancy unit of Erasmus MC University Medical Center (Erasmus MC) will be assessed for eligibility. LUMC is the coordinating centre. Couples with RPL will be invited to participate at their intake visit (after they have been referred by their general practitioner or a referring hospital). After diagnostic investigations on known risk factors of RPL are completed, couples with unexplained RPL will be selected for inclusion. In addition, couples who visited the participating clinics in the period 2012-2019 will be included in retrospect. Couples with RPL will participate in both the case-control study and the cohort study.

Eligible couples visiting the antenatal outpatient clinic of LUMC during their pregnancy will be invited to participate in the control group. Control couples will also be included in retrospect.

Study recruitment in the coordinating centre started in June 2019. Recruitment at Erasmus MC is expected to start in September 2019. All couples will receive written information about the study together with the informed consent form, which includes a request to obtain permission for gathering data from medical records and storage of biomaterial for additional analyses related to this study. Participants are informed that study participation is voluntary and that they are free to withdraw at any time without any consequences for subsequent care. In case of participation, the informed consent form should be signed prior to inclusion in the study.

\section{Study procedures}

\section{Collection of clinical characteristics}

Data about obstetric and general medical history and lifestyle factors of all participating couples will be documented (table 1).

These data will be collected during consultations (in a semistandardised way using a template) and from medical records. Additional required data will be acquired via digital surveys that will be sent to participating couples. Data entry and generation of digital surveys will be performed using Castor EDC. ${ }^{50}$

Couples with RPL participating in the cohort study will be in follow-up for a total time of 5 years after initial consultation. These couples will receive a digital survey once a year. This survey contains questions about outcomes of new pregnancies if applicable and changes in medical history and lifestyle in the past year. When couples with RPL are still in regular clinical follow-up, data will be collected during regular consultations and it will not be necessary to send a digital survey. Retrospectively included couples from whom (part of) the follow-up period is missing in their medical records, will receive a survey to ask for pregnancy outcomes in the missing time period.

Clinical characteristics of couples participating in the control group will be collected at one time point (during consultation at the antenatal clinic), directly followed by a digital survey containing questions about lifestyle related to the period prior to the index pregnancy. There is no follow-up of control couples.

\section{Collection and analysis of samples}

Male partners of participating couples will be asked for a peripheral blood sample and sperm sample acquired through masturbation. Samples will be collected from all

\section{Table 1 Collection of data}

\begin{tabular}{|c|c|}
\hline & Parameters \\
\hline $\begin{array}{l}\text { Maternal } \\
\text { characteristics }\end{array}$ & $\begin{array}{l}\text { Date of birth, zip code, ethnicity, level of education, profession, body weight, height, general medical } \\
\text { history, use of medication, family history, detailed obstetric history (parity, no of spontaneous pregnancy } \\
\text { losses, ectopic pregnancies or induced abortions, modes of conception of previous births, modes } \\
\text { of delivery of previous births, gestational age at previous births, complications during previous } \\
\text { pregnancies and deliveries, birth weight, gender and Apgar score of children of previous births), lifestyle } \\
\text { characteristics (smoking, alcohol, drugs and caffeine intake, physical exercise pattern). }\end{array}$ \\
\hline $\begin{array}{l}\text { Results of (previous) } \\
\text { investigations into } \\
\text { known risk factors } \\
\text { of RPL }\end{array}$ & $\begin{array}{l}\text { Presence of antiphospholipid syndrome (anticardiolipin IgG and IgM, B2 glycoprotein I antibodies IgG } \\
\text { and IgM, and lupus anticoagulans), parental chromosomal abnormalities, presence of thyroid antibodies, } \\
\text { presence of uterine anomalies. }\end{array}$ \\
\hline
\end{tabular}

RPL, recurrent pregnancy loss. 
prospectively included men. This applies to both cases and controls. From all retrospectively included couples, only clinical data will be documented.

All samples will be processed and analysed in the laboratory of Reproductive Immunology at LUMC. Samples will be collected once. Samples from other participating centres will be sent to LUMC for storage and analysis.

Semen samples will be stored in $-20^{\circ} \mathrm{C}$ until time of analysis. Sperm DNA fragmentation will be detected by terminal deoxynucleotidyltransferase dUTP nick end labelling assay (APO-DIRECT Kit, BD Biosciences) following the manufacturer's instructions. The level (\%) of sperm DNA fragmentation will be determined by flow cytometric analysis.

The level of immunomodulatory factors in seminal plasma and peripheral blood will be assessed by BioPlex Luminex system assay (Bio-Rad Laboratories), following the manufacturer's instructions. Samples will be analysed using a Bio-Plex Array Reader with Bio-Plex software. Through this assay quantification of cytokine levels including tumour necrosis factor- $\alpha$, IFN- $\gamma$, transforming growth factor- $\beta 1$, IL-1 $\beta$, IL-8, IL-10, IL-11, IL-18, sHLA-G and PGE2 will be performed. These cytokines were selected because previous small studies suggested correlations between concentrations in seminal plasma and/or paternal peripheral blood and reproductive outcome. ${ }^{47-49} 51$

\section{Control of bias}

Since the design of this study is observational, there is need to control and adjust for confounding factors. For example, maternal age is an important confounder for the effect of paternal age on RPL. To control for confounders, stratification and regression models will be used. Selection bias is minimised by a clear definition of the study population. In addition, the control couples are selected independently of their exposure and they represent the source population that generates the cases. Finally, information bias is limited as much as possible by collecting information similarly from the cases and controls.

\section{Sample size calculation \\ Case-control study}

Since sperm DNA fragmentation could be seen as a proxy for advanced age and also for the presence of smoking, obesity and excessive exercise, this factor was used for sample size calculations. Zhao et $a l^{40}$ evaluated the association between sperm DNA fragmentation and miscarriages after IVF/ICSI treatment in 2756 couples and they found a combined OR 2.28 (95\% CI 1.55 to 3.35 ) for miscarriage in patients with high sperm DNA fragmentation The rate of high sperm DNA fragmentation was significantly higher in the group with miscarriage (34\%) compared with the group with live births (19\%). To detect this difference, using $\alpha=0.05$ and power $=80 \%$, the sample size would be 135 in the RPL group and 135 in the control group. Also the recent meta-analyses of Tan $e t a t^{41}$ and McQueen $e t a t^{42}$ have been taken into consideration for sample size calculation. They evaluated the mean difference in per cent sperm DNA fragmentation between RPL patients and fertile controls. However, based on these mean differences (both of approximately $12 \%)$, the sample size would be very small $(<10$ per arm) and therefore not appropriate for this project, since we are not solely interested in sperm DNA fragmentation but also in other lifestyle and demographic factors.

\section{Cohort study}

No straightforward accepted methods exist to estimate the required number of subjects to develop a multivariable prediction model. Ideally, prognostic studies include several hundreds of patients who develop the outcome event. ${ }^{52}$ Various studies have suggested that for each candidate predictor studied, at least 10 events are required. ${ }^{53} 54$ Currently, female age and number of previous pregnancy losses are the only known factors consistently shown to impact prognosis for future pregnancy outcomes. ${ }^{1}$ In addition to these factors, we intend to examine paternal factors for their predictive capacity. Assuming that at least two paternal factors will be included in the model, like age and BMI (and also maternal BMI), with four age categories $(<30,30-35,35-40,>40$ years $)$, four categories for preceding pregnancy losses $(2,3,4, \geq 5)$ and four BMI categories $\left(<18,18-25,25-30,>30 \mathrm{~kg} / \mathrm{m}^{2}\right)$, a minimum of $20 \times 10=200$ patients with RPL and live birth in subsequent pregnancy are necessary. We estimate that the total RPL cohort will eventually consist of approximately 735 couples (with retrospective and prospective inclusions together, shown in figure 1) and we expect $70 \%$ of them to have a live birth within 5 years after initial consultation. Based on these numbers, it is feasible to develop a multivariable model to predict the chances for ongoing pregnancy and live birth within 5 years. We will include patients who visited the clinics between 2012 and 2019 and also the couples (cases) of the case-control study.

\section{Study outcomes}

In the case-control study, the following exposures will be studied:

- Smoking: documented as average number of cigarettes per day. Also data on former smoking behaviour will be documented.

- Alcohol consumption: documented as average number of units per week.

- Recreational drug consumption: specified by type of drug, quantity and frequency.

- Caffeine intake: documented as average number of caffeinated drinks per day.

- Physical exercise pattern; documented as moderate to intensive physical exercise in days per week and minutes per day.

In the cohort study, the following outcomes will be studied:

- Live birth within 5 years after initial consultation (for this outcome we intend to develop a prediction model). 
- Ongoing pregnancy (>24weeks).

- Time interval until next pregnancy.

- Pregnancy complications including fetal growth restriction, preterm birth, pregnancy induced hypertension, pre-eclampsia, haemolysis elevated liver enzymes and low platelets syndrome and gestational diabetes mellitus.

\section{Statistical analysis plan}

\section{Case-control study}

For the case-control study, proportions will be calculated for the dichotomous and categorial exposures with 95\% CIs. Comparison between the cases and controls is performed by a $\mathrm{X}^{2}$ test. Mean differences with $95 \%$ CIs are calculated to compare continuous variables between the groups. To correct for confounders (including maternal factors), stratified analyses and multivariate logistic regression including paternal and maternal variables that are highly correlated will be performed.

\section{Cohort study}

To indicate a relation between live birth and paternal (and maternal) factors as described above, first univariate logistic regression will be used. To select the most prognostic set of variables logistic regression with shrinkage methods such as lasso will be used. Time to pregnancy is estimated using the Kaplan-Meier method. Only in the subgroup of prospectively included RPL couples (with collection of samples), blood and sperm investigations will be included in the analyses.

To cope with analysis of missing values, multiple imputation will be performed. Statistical analysis will be performed using SPSS Statistics V.25 (IBM SPSS Software) and/or R V.3.6.0. For all tests, a two-sided $\mathrm{p}<0.05$ or $95 \%$ CI not including the null value is considered significant.

\section{Patient and public involvement}

During the development of the study protocol, the Dutch association for patients with fertility problems (Freya) was consulted. Results will be presented during their thematic meetings to inform patients about study progress. Social media will be used to highlight new publications and conference presentations.

\section{ETHICS AND DISSEMINATION}

This study will be conducted according to the principles of the Declaration of Helsinki. ${ }^{55}$ Ethics approval for this study was obtained at the Medical Research Ethics Committee of the Leiden University Medical Center. No risks or burden are expected from the study. No additional hospital visits are required.

Eligible couples obtain written information about the study objectives and procedures and they will have sufficient time to decide on participating. All clinical data and data derived from surveys will be saved in the Castor EDC REMI III database. No data directly traceable to patients will be included in this database. Every couple will be assigned a unique code. This code will also be used to associate clinical data with corresponding blood and semen samples.

The findings of this study will be disseminated via peerreviewed publications and presentations at international conferences.

\section{DISCUSSION}

RPL is often accompanied by psychological morbidities such as depression and anxiety, making it a very distressing and costly condition. ${ }^{56}$ In current practice, RPL is mostly considered an issue derived exclusively from female causes. However, it is questionable whether this female-centred approach is correct, especially considering the substantial proportion of RPL cases that remains unexplained. In November 2017, the ESHRE developed a new guideline for the management of RPL, to supply healthcare providers with the best available evidence for investigation and treatment of RPL. Future research on the paternal contribution in RPL, such as the impact of paternal lifestyle factors and sperm DNA damage, was recommended by the guideline committee. ${ }^{1}$

In this project, we hypothesise that besides maternal factors, paternal factors are associated with the development of RPL. Understanding the role of these factors contributing to the pathological mechanisms of RPL may provide new diagnostic tools and treatment options. To the best of our knowledge, this project includes the first large prospective cohort study evaluating the contribution of multiple paternal lifestyle and biological factors to unexplained RPL.

Limitations of all research on lifestyle factors using selfreported data are the phenomena of recall and response bias. Individuals might report biassed estimates of selfassessed behaviour for different reasons, including misunderstanding or social desirability. Although these types of bias will always be present to some extent, we try to minimise this by using standardised and well-structured surveys, by avoiding long recall periods as much as possible and by choosing an appropriate and well-defined control group.

Ultimately, we aim to develop a couple-specific model including both maternal and paternal factors to predict future reproductive outcomes in couples with unexplained RPL. Although not an intervention as such, counselling couples confronted with RPL about their individual prognosis is an essential part of the management of these couples and allows them to decide for or against future pregnancy attempts. Moreover, this study might also provide new starting points for future treatment options with regard to lifestyle interventions.

\section{Author affiliations}

${ }^{1}$ Gynaecology and Obstetrics, Leiden University Medical Center, Leiden, The Netherlands

${ }^{2}$ Immunohematology and Blood Transfusion, Leiden University Medical Center, Leiden, The Netherlands

${ }^{3}$ Clinical Epidemiology, Leiden University Medical Center, Leiden, The Netherlands 
${ }^{4}$ Biomedical Data Sciences, Leiden University Medical Center, Leiden, The Netherlands

${ }^{5}$ Gynaecology and Obstetrics, Erasmus Medical Center, Rotterdam, The Netherlands

Contributors EL and M-LvdH drafted the protocol and then NdF wrote the protocol in accordance with the coauthors' contributions. ME and SH complemented on the immunological questions in this protocol and SIC, JVL and AM improved the methodological aspects. All authors contributed to the writing and reviewing of this article and gave final approval of the version to be published.

Funding This project has funding from the Leiden University research profile 'Innovation in Health Strategy and Quality of Care'.

Competing interests None declared.

Patient consent for publication Not required.

Provenance and peer review Not commissioned; externally peer reviewed.

Open access This is an open access article distributed in accordance with the Creative Commons Attribution Non Commercial (CC BY-NC 4.0) license, which permits others to distribute, remix, adapt, build upon this work non-commercially, and license their derivative works on different terms, provided the original work is properly cited, appropriate credit is given, any changes made indicated, and the use is non-commercial. See: http://creativecommons.org/licenses/by-nc/4.0/.

ORCID iD

Nadia du Fossé http://orcid.org/0000-0002-1952-3608

\section{REFERENCES}

1 ESHRE Early Pregnancy Guideline Development Group. Recurrent Pregnancy Loss. Guideline of the European Society of Human Reproduction and Embryology, 2017.

2 Nybo Andersen AM, Wohlfahrt J, Christens P, et al. Maternal age and fetal loss: population based register linkage study. BMJ 2000;320:1708-12.

3 Kolte AM, Bernardi LA, Christiansen OB, et al. Terminology for pregnancy loss prior to viability: a consensus statement from the ESHRE early pregnancy special interest group. Human Reproduction 2015;30:495-8.

4 Rai R, Regan L. Recurrent miscarriage. Lancet 2006;368:601-11.

5 Jauniaux E, Farquharson RG, Christiansen OB, et al. Evidence-Based guidelines for the investigation and medical treatment of recurrent miscarriage. Hum Reprod 2006;21:2216-22.

6 Miyakis S, Lockshin MD, Atsumi T, et al. International consensus statement on an update of the classification criteria for definite antiphospholipid syndrome (APS). J Thromb Haemost 2006:4:295-306.

7 van den Boogaard E, Cohn DM, Korevaar JC, et al. Number and sequence of preceding miscarriages and maternal age for the prediction of antiphospholipid syndrome in women with recurrent miscarriage. Fertil Steril 2013;99:188-92.

8 Woelfer B, Salim R, Banerjee S, et al. Reproductive outcomes in women with congenital uterine anomalies detected by three-dimensional ultrasound screening. Obstet Gynecol 2001:98:1099-103.

9 Salim R, Woelfer B, Backos M, et al. Reproducibility of threedimensional ultrasound diagnosis of congenital uterine anomalies. Ultrasound Obstet Gynecol 2003;21:578-82.

10 Lata K, Dutta P, Sridhar S, et al. Thyroid autoimmunity and obstetric outcomes in women with recurrent miscarriage: a case-control study. 2013;2:118-24

11 Hook EB, Healy NP, Willey AM. How much difference does chromosome banding make?: adjustments in prevalence and mutation rates of human structural cytogenetic abnormalities. Ann Hum Genet 1989;53:237-42.

12 Braekeleer MD, Dao T-N. Cytogenetic studies in couples experiencing repeated pregnancy losses. Hum Reprod 1990;5:519-28.

13 Goddijn M, Leschot NJ. Genetic aspects of miscarriage. Best Pract Res Clin Obstet Gynaecol 2000;14:855-65.

14 Gardella JR, Hill III JA. Environmental toxins associated with recurrent pregnancy loss. Semin Reprod Med 2000;18:407-24.

15 Christiansen OB, Nybo Andersen A-M, Bosch E, et al. EvidenceBased investigations and treatments of recurrent pregnancy loss. Fertil Steril 2005;83:821-39.

16 George L, Granath F, Johansson ALV, et al. Risks of repeated miscarriage. Paediatr Perinat Epidemiol 2006;20:119-26.
17 Lashen H, Fear K, Sturdee DW. Obesity is associated with increased risk of first trimester and recurrent miscarriage: matched case-control study. Hum Reprod 2004;19:1644-6.

18 Cnattingius S, Signorello LB, Annerén G, et al. Caffeine intake and the risk of first-trimester spontaneous abortion. $N$ Engl J Med 2000;343:1839-45.

19 George L, Granath F, Johansson AL, et al. Environmental tobacco smoke and risk of spontaneous abortion. Epidemiology 2006;17:500-5.

20 Stephenson MD. Frequency of factors associated with habitual abortion in 197 couples. Fertil Steril 1996;66:24-9.

21 Jaslow CR, Carney JL, Kutteh WH. Diagnostic factors identified in 1020 women with two versus three or more recurrent pregnancy losses. Fertil Steril 2010;93:1234-43.

22 Liddell HS, Pattison NS, Zanderigo A. Recurrent Miscarriage Outcome After Supportive Care in Early Pregnancy. Aust N Z J Obstet Gynaecol 1991;31:320-2.

23 Lund M, Kamper-Jorgensen M, Nielsen HS, et al. Prognosis for live birth in women with recurrent miscarriage: what is the best measure of success? Obstet Gynecol 2012;119:37-43.

24 Kaandorp SP, Goddijn M, van der Post JAM, et al. Aspirin plus heparin or aspirin alone in women with recurrent miscarriage. $N$ Engl J Med 2010;362:1586-96.

25 Badawy AM, Khiary M, Sherif LS, et al. Low-Molecular weight heparin in patients with recurrent early miscarriages of unknown aetiology. J Obstet Gynaecol 2008;28:280-4.

26 Fawzy M, Shokeir T, El-Tatongy M, et al. Treatment options and pregnancy outcome in women with idiopathic recurrent miscarriage: a randomized placebo-controlled study. Arch Gynecol Obstet 2008;278:33-8.

27 Sugiura-Ogasawara M, Ozaki Y, Kitaori T, et al. Original article: live birth rate according to maternal age and previous number of recurrent miscarriages. Am J Reprod Immunol 2009;62:314-9.

28 de La Rochebrochard E, Thonneau P. Paternal age and maternal age are risk factors for miscarriage; results of a multicentre European study. Hum Reprod 2002;17:1649-56.

29 Venners SA, Wang X, Chen C, et al. Paternal smoking and pregnancy loss: a prospective study using a biomarker of pregnancy. Am J Epidemiol 2004;159:993-1001.

30 Colaci DS, Afeiche M, Gaskins AJ, et al. Men's body mass index in relation to embryo quality and clinical outcomes in couples undergoing in vitro fertilization. Fertil Steril 2012;98:1193-9.

31 Sbracia M, Cozza G, Grasso JA, et al. Semen parameters and sperm morphology in men in unexplained recurrent spontaneous abortion, before and during a 3 year follow-up period. Hum Reprod 1996;11:117-20.

32 Gopalkrishnan K, Padwal V, Meherji PK, et al. Poor quality of sperm as it affects repeated early pregnancy loss. Arch Androl 2000;45:111-7.

33 Bhattacharya SM. Association of various sperm parameters with unexplained repeated early pregnancy loss - which is most important? Int Urol Nephrol 2008;40:391-5.

34 Brahem S, Mehdi M, Landolsi $\mathrm{H}$, et al. Semen parameters and sperm DNA fragmentation as causes of recurrent pregnancy loss. Urology 2011;78:792-6

35 Imam SN, Shamsi MB, Kumar K, et al. Idiopathic recurrent pregnancy loss: role of paternal factors; a pilot study. J Reprod Infertil 2011;12:267-76

36 Talebi AR, Vahidi S, Aflatoonian A, et al. Cytochemical evaluation of sperm chromatin and DNA integrity in couples with unexplained recurrent spontaneous abortions. Andrologia 2012;44:462-70.

37 Khadem N, Poorhoseyni A, Jalali M, et al. Sperm DNA fragmentation in couples with unexplained recurrent spontaneous abortions. Andrologia 2014;46:126-30.

38 Zhang L, Wang L, Zhang X, et al. Sperm chromatin integrity may predict future fertility for unexplained recurrent spontaneous abortion patients. Int J Androl 2012;35:752-7.

39 Robinson L, Gallos ID, Conner SJ, et al. The effect of sperm DNA fragmentation on miscarriage rates: a systematic review and metaanalysis. Human Reproduction 2012;27:2908-17.

40 Zhao J, Zhang Q, Wang Y, et al. Whether sperm deoxyribonucleic acid fragmentation has an effect on pregnancy and miscarriage after in vitro fertilization/intracytoplasmic sperm injection: a systematic review and meta-analysis. Fertil Steril 2014:102:998-1005.

41 Tan J, Taskin O, Albert A, et al. Association between sperm DNA fragmentation and idiopathic recurrent pregnancy loss: a systematic review and meta-analysis. Reprod Biomed 2018.

42 McQueen DB, Zhang J, Robins JC. Sperm DNA fragmentation and recurrent pregnancy loss: a systematic review and meta-analysis. Fertil Steril 2019. 
43 Moldenhauer LM, Diener KR, Thring DM, et al. Cross-Presentation of male seminal fluid antigens elicits T cell activation to initiate the female immune response to pregnancy. J Immunol 2009;182:8080-93.

44 Robertson SA. Seminal plasma and male factor signalling in the female reproductive tract. Cell Tissue Res 2005;322:43-52.

45 Meuleman T, Snaterse G, van Beelen E, et al. The immunomodulating effect of seminal plasma on T cells. J Reprod Immunol 2015;110:109-16.

46 Nederlof I, Meuleman T, van der Hoorn MLP, et al. The seed to success: the role of seminal plasma in pregnancy. $J$ Reprod Immunol 2017;123:24-8

47 Nikolaeva MA, Babayan AA, Stepanova EO, et al. The relationship of seminal transforming growth factor- $\beta 1$ and interleukin- 18 with reproductive success in women exposed to seminal plasma during IVF/ICSI treatment. J Reprod Immunol 2016;117:45-51.

48 Seshadri S, Bates M, Vince G, et al. Cytokine expression in the seminal plasma and its effects on fertilisation rates in an IVF cycle. Andrologia 2011;43:378-86.

49 Havrylyuk A, Chopyak V, Boyko Y, et al. Cytokines in the blood and semen of infertile patients. Cejoi 2015;3:337-44.
50 Ciwit BV. Castor Electronic Data Capture [Internet], 2018.

51 Larsen MH, Bzorek M, Pass MB, et al. Human leukocyte antigen-G in the male reproductive system and in seminal plasma. Mol Hum Reprod 2011:17:727-38.

52 Moons KGM, Royston P, Vergouwe Y, et al. Prognosis and prognostic research: what, why, and how? BMJ 2009;338:b375

53 Laupacis A, Sekar N, Stiell IG. Clinical prediction rules. A review and suggested modifications of methodological standards. JAMA 1997;277:488-94.

54 Harrell FE, LEE KL, Mark DB. Multivariable prognostic models: issues in developing models, evaluating assumptions and adequacy, and measuring and reducing errors. Stat Med 1996;15:361-87.

55 World Medical Association Declaration of Helsinki. Ethical principles for medical research involving human subjects. JAMA 2013;310:2191-4.

56 Serrano F, Lima ML. Recurrent miscarriage: psychological and relational consequences for couples. Psychol Psychother 2006;79:585-94. 